\title{
Multi-Partite Structure of Demand-Supply Network Element
}

\author{
I. Fekete ${ }^{1}$, G. Kallós ${ }^{2}$, T. Hartványi ${ }^{3}$ \\ Széchenyi István University \\ Egyetem tér 1, 9026, Győr, Hungary \\ Phone: +36 207770243 \\ e-mail: ${ }^{1}$ fekete.istvan.pic@gmail.com, ${ }^{2}$ kallos@sze.hu, ${ }^{3}$ hartvanyi@sze.hu
}

\begin{abstract}
In our recent article we investigate the utilization possibilities of usual time related, demand data sets of an organization. We also create some derivative data sets from those time related ones, thanks to what we have the full set of links' and nodes' data for further matrix analyses. We also evaluate some usual network analytical approaches to determine the characteristic structure of demand-supply network element (i.e. organization), the relevant network topology and sensitive to failure network skeleton.
\end{abstract}

Keywords: supply chain, multipartite networked structures, demand-supply data, matrix analysis

\section{Introduction}

In the last years we have developed a visual, low IT-profile, low-cost analytical approach for investigating the network topology of an organization, i.e. the Excel-based Combined Quantity Irregularity Graphs [5]. That CQIG approach is powerful, effective and efficient in case of smaller organizations, where the number of nodes (Suppliers, Resources, Finished Products and Customers) are in the range of hundreds to a thousand. Even in such organization the number of related links falls in the range of several thousands. Such complexity is easily handled by our CQIG approach, where we can define the network-skeleton-building nodes and links, and focusing on those few, we can increase the resilience of our organization considerably, more than just using the classical supply chain heuristics. Our CQIG approach has its limitations, especially of large organizations with thousands of nodes and of complex nested structure. Therefore, we have started to work out a still low-IT and low-investment profile solution based on matrices. First we evaluate the usual time related demand data sets of an organization in network context. Simultaneously, we define what kind of data are to be used to create the derivatives to build the required matrices for network analytical investigations. Then we introduce the different matrix-representation of the networked structure of an organization. Our approach focuses on networked structure of demand-supply network 
(DSN), and is not competing to those well based solutions like Theory of Constraints [7], Toyota Way [8], Multi-Echelon Inventory Optimization [11] but complementing to them.

\section{Material conversion and transport route in DSN}

Before we start investigating the data and matrix representation, there is a special aspect of the Demand-Supply Networks to be thoroughly understood. The material conversion and transport process shows strong analogy to the irreversible metabolic chemical reactions. The man and machine in such material conversion plays the enabler's role similar to enzymes of metabolic reactions or molecular chaperones. The substrate proteins of interactomes (protein-protein interaction network) correspond to materials (to be converted in an organization). The chaperone proteins forming monooligomers are analogous to machines of different complexity, what can also be grouped in a production line or cell. Those proteins which are forming hetero-oligomers are more analogous to humans in their interactions. The biochemical network achieves the perfection through frequent repair of the damaged products, differing from the right quality first time approach in production. $[3,4]$.

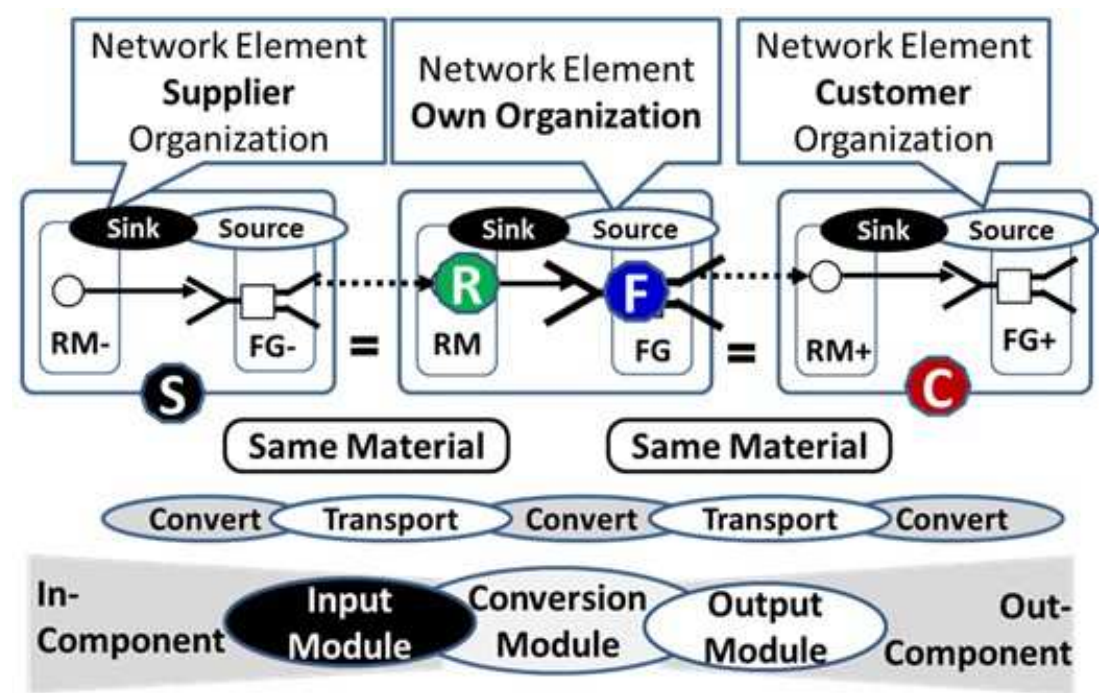

Figure 1. Material conversion and transport route in DSN

In case of material conversion and transport route in DSN. The Make-process of SCOR Model [12] converts the raw material resources (Fig. 1: RM, R-node) from Input Module into new material form, i.e. finished products (Fig. 1: FG, F-node). In the Source- and Deliver-processes the same material is travelling - transport in network terminology. On the other hand, the same material form is having different coding at the different organizations due to the differences in ERP-systems of the organizations, and because of the fact that the output (finished product) of the supplier is the input (raw 
material) of the subsequent customer. Consequently, with S-R-F-C paths we cover the material conversion and transport routes, what, in turn, are the building patterns of Demand-Supply Network. See Fig. 1. Further explanation on networked structure of the Conversion Module in an organization you can find in our previous publication [6].

\section{Matrix model of the Demand-Supply Network element}

In our previous publication we have described the inter-relationships between supply chain and network terminologies in details, as well as the main structural characteristics in and around an organization - Demand-Supply Network Element [6]. Here we restrict our attention to a simplified network model of an organization with different level of granularity for internal and for external structural representation. Concerning our organization's internal structure, we focus on modules (more densely connected network parts) and the structure of network skeleton - high importance/high-centrality nodes: hubs, bridges, and high weight links $[3,9,10]$.

The matrix method enables us to analyse the entire network of an organization simultaneously, including the supplier- and customer nodes as well, and modelling organizations with complex modular structure. It opens the way for modelling the failure propagation as well. As we already discussed, usually we have information and data about an organization's internal structure at very detailed level (fine-grained representation). Regarding to organization's external structure, the links with the organization's suppliers and customers are well known, while the finer-grained structure of the suppliers or customers are usually poorly known to us. Therefore, when analysing our organization's external structure, we suggest to consider the customers and suppliers as nodes (network elements of DSN) grouped in echelons. The nodes from our organization are linked to those network elements, rather than to the resource/finished product nodes of unknown to us structure in supplier/customer organizations respectively (Fig. 2).

Because of the large number of different matrices and symbols defined in this section we use the following simplified notation for matrix $\mathbf{A}$ of size $\mathbf{s} \times \mathbf{r}: \mathbf{A}(\mathbf{s r})=\left(a_{i j}\right) \epsilon \mathbb{R}^{\mathrm{s} \times \mathbf{r}}$. The entry of that matrix $\mathbf{A}$ will be noted as $A[s, r]$ instead of commonly used form of $a_{i j}$. In both cases the capital letter (sometimes with lower case letters of additional information) defines the entry type - like $\mathbf{A}$ for adjacency matrix, $\mathbf{C}_{\mathbf{b c}}$ for betweenness centrality matrix. The difference to Newman's notation is that $\left(a_{i j}\right)$ represents a directed link from node $i$ to node $j$, as it is usual in the Hungarian notations $[1,9]$.

According to our network model of an organization, 4 echelons of nodes are to be investigated: echelon of suppliers $\left(S_{1}-S_{s}\right)$, echelon of resources $\left(R_{1}-R_{r}\right)$, echelon of finished products $\left(\mathrm{F}_{1}-\mathrm{F}_{\mathrm{f}}\right)$ and echelon of customers $\left(\mathrm{C}_{1}-\mathrm{C}_{\mathrm{c}}\right)$. As we already highlighted, in case of suppliers and customers we neglect their internal structure. In Fig. 2 a simple networked structure of an organization and its matrix representation are shown on the left, where the materials flow only in one direction, from suppliers to customers, through material conversion and transport process, i.e. directed acyclic graph or DAG in the literature [9]. The adjacency matrix $\mathbf{A}(\mathbf{n n})$ of such organization model is a quadratic upper-triangle strictly nil-potent matrix (no self-edges, no reverse flow), containing three rectangular adjacency matrices of $\mathbf{A}(\mathbf{s r}), \mathbf{A}(\mathbf{r f})$, and $\mathbf{A}(\mathbf{f} \mathbf{c})$ for the 
adjacent echelon pairs - where $\mathbf{n}=\mathbf{s}+\mathbf{r}+\mathbf{f}+\mathbf{c}$ is the size of the represented network. The values in the adjacency matrix are either 0 (no link) or 1 (linked). Such basic uppertriangle quadratic matrix representation is suitable for analysing the path length characteristics in our network, for stratification through creating the bibliographic coupling and co-citation matrices [9] and calculating the betweenness centralities as well. Evidently, if the organization model is having additional echelon, then that additional echelon will form further rectangular block in our matrix with its relevant adjacent echelon pair.
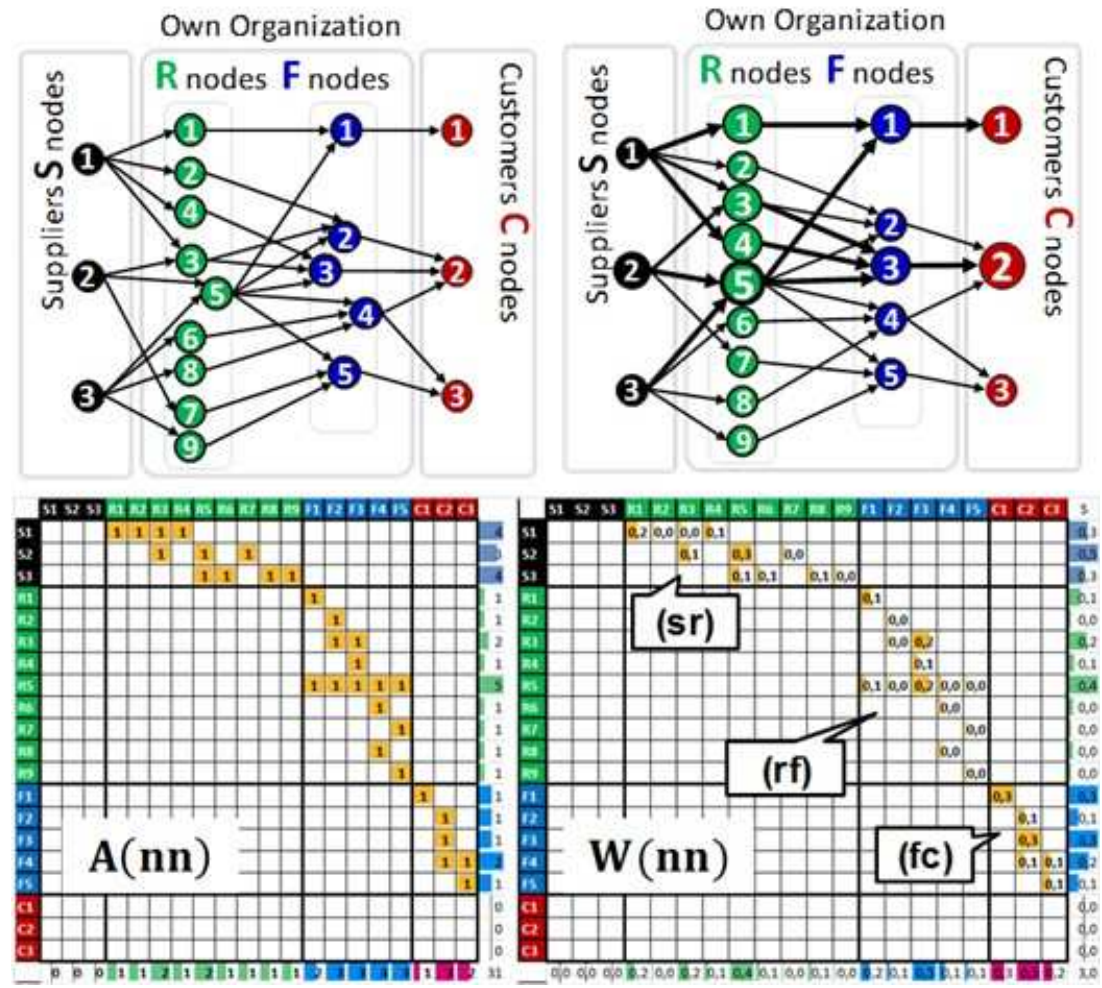

Figure 2. Network model of an organization. Top-left graph and its matrix representation below are without weight, while on the right are with weight.

The nodes and links may have weights $[3,9]$ in the network representations. That has pivotal role in differentiating the so-called weak-links from strong links. While the latter links determine the network skeleton, the former links contribute to network's stability in several ways [3]. The top right network and the matrix $\mathbf{W}(\mathbf{n n})$ below it in Fig. 2 models a weighted network of the same structure. The column graphs at the right side and bottom side of the matrices visualize the out-weight and in-weight of each node in its echelon respectively. 


\section{Data sets of an organization and the created derivatives}

In all businesses we can get the time dependent demand data according to Source-, Make-, Deliver-processes of the SCOR model [12]. For each echelon pair of $\{\mathbf{s r}\},\{\mathbf{r f}\}$ and $\{\mathbf{f c}\}$ we collect the time dependent variables generating the entries of $D[t, i, j]$, where $i$ stands for the node from which the link goes to the node $j$ and $t$ differentiates the time slots (see Fig. 3). The historical material arrivals from our suppliers determine the links between the supplier- and resource nodes $-D[t, s, r]$. The historical finished product deliveries to our customers determine the links between the finished productand customer nodes $-D[t, f, c]$. The historical material consumption in finished products determines the links between the resource nodes and the finished product nodes $-D[t, r, f]$. That can be either downloaded as backflush data from MRP (material replenishment planning) system or calculated through multiplying the production volumes of the finished product node with its Bill-of-Material (BoM).

We generate the link related derivatives for each echelon pair of $\{\mathbf{s r}\},\{\mathbf{r f}\}$ and $\{\mathbf{f c}\}$ separately according to the Equations of 3-1 till 3-4 in Fig. 3. From time dependent variables $D[t, i, j]$ we calculate the mean $D_{\mu}[i, j]$ for each link in the echelon pair (Eq. 31). $D_{\mu}[i, j]$ serves for calculating the adjacency $A[i, j]$ through Equation 3-2 and the weight of the link $W[i, j]$ as per the Equation 3-3. The time dependent variables $D[t, i, j]$ and the mean $D_{\mu}[i, j]$ deliver the standard deviation $D_{\sigma}[i, j]$ of the links between the nodes of the echelon pair through Equation 3-4.

The Equations 3-5 till 3-7 in Figure 3 determine the different types of irregularities of the nodes. In matrix analytical method we use echelon level normalization to calculate a node's irregularity both in destabilized and normal (calm) modes. Such irregularity I call meta-level irregularity and are denoted as $M_{D}[i]$ and $M_{C}[i]$ respectively (see Equations 3-5 and 3-6). The destabilized and normal modes correspond to distributed VS centralised demand pooling, or correlated VS non-correlating demands [11]. The Equation 3-7 $I[i]$ delivers similar to CQIG-irregularity of the node according to its outlinks' behaviour [5]. However, the meta-level irregularity is more reasonable to use, since the meta-level irregularity of a node underlines its contribution to the network's perturbation. Similar meta-level normalization of the standard deviation is done at the analyses of food webs, which are also directed networks, just the in-links of the predators (users of the resource preys) are not in strong sync, but behave as alternative in-links [9]. Furthermore, there is a high potential in simulating the failure dispersion through modelling the switch of a node's state from normal (calm) to destabilized and contributing to the load of the nodes neighbouring the destabilized one through switching from $M_{C}[i]$ to $M_{D}[i]$.

Please note: when including the man-nodes and machine-nodes in the resource echelon as well, the normalization of the different resource types (3M) must be carried out separately within the cluster of that type - e.g. man-node by the echelon-level mean of man-nodes, etc. 


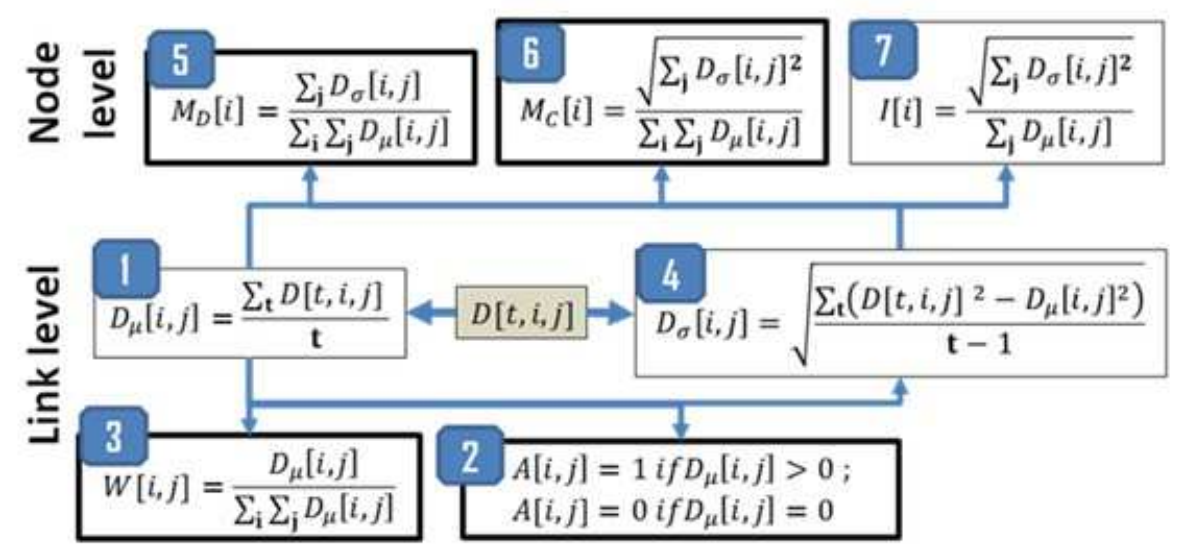

$D[t, i, j]$ : link's time dependent demand variable $D_{\mu}[i, j]$ : mean of link's demand $A[i, j]$ : link's existence $W[i, j]$ : link's weight $D_{\sigma}[i, j]$ : std. deviation of link's demand $M_{D}[i]$ : meta-level irregularity of destabilized node $I[i]$ : node's irregularity $M_{C}[i]$ : meta-level irregularity of stable (calm) node $\mathbf{t}$ : number of time periods

$$
\begin{array}{ll}
i \epsilon[1,2, \ldots \mathbf{s}] \text { and } j \epsilon[1,2, \ldots \mathbf{r}] \text { or } & \mathbf{s}: \text { number of suppliers } \\
i \epsilon[1,2, \ldots \mathbf{r}] \text { and } j \epsilon[1,2, \ldots \mathbf{f}] \text { or } & \mathbf{r} \text { : number of resources } \\
i \epsilon[1,2, \ldots \mathbf{f}] \text { and } j \epsilon[1,2, \ldots \mathbf{c}] \text { where } & \mathbf{f} \text { : number of finished products } \\
& \mathbf{c} \text { : number customers }
\end{array}
$$

Figure 3. Equations to create the data sets of matrices for network analyses of an organization

\section{Matrices for defining the stratification and the network skeleton of the organization}

Fig. 4 show the matrices we create from the above determined data for analysing the network structure and networked behaviour of an organization. Building the matrices of 4-1 till 4-7 is possible either by non-weighted adjacency matrices $\mathbf{A}(\mathbf{f} \mathbf{c}), \mathbf{A}(\mathbf{r f}), \mathbf{A}(\mathbf{s r})$ or by their weighted pairs $\mathbf{W}(\mathbf{f c}), \mathbf{W}(\mathbf{r f}), \mathbf{W}(\mathbf{s r})$ denoted as 8 in Fig. 4. Equation 4-1 shows how we build the quadratic matrix $\mathbf{A}(\mathbf{n n})$ from the rectangular block matrices.

The connectedness within the organization is not evenly dense. The longitudinal dimension of the modularization is accountable for the echelons, which are already well modelled by our quadratic matrix with rectangular blocks of echelon-pairs, while the transversal dimension of the modularization is responsible for stratification of the DSN. That latter is manifested in value streams of an organization. The stratification may be latent, but to our luck, those transversal interdependences can be well understood through the combined bi-fan and bi-parallel motifs. 


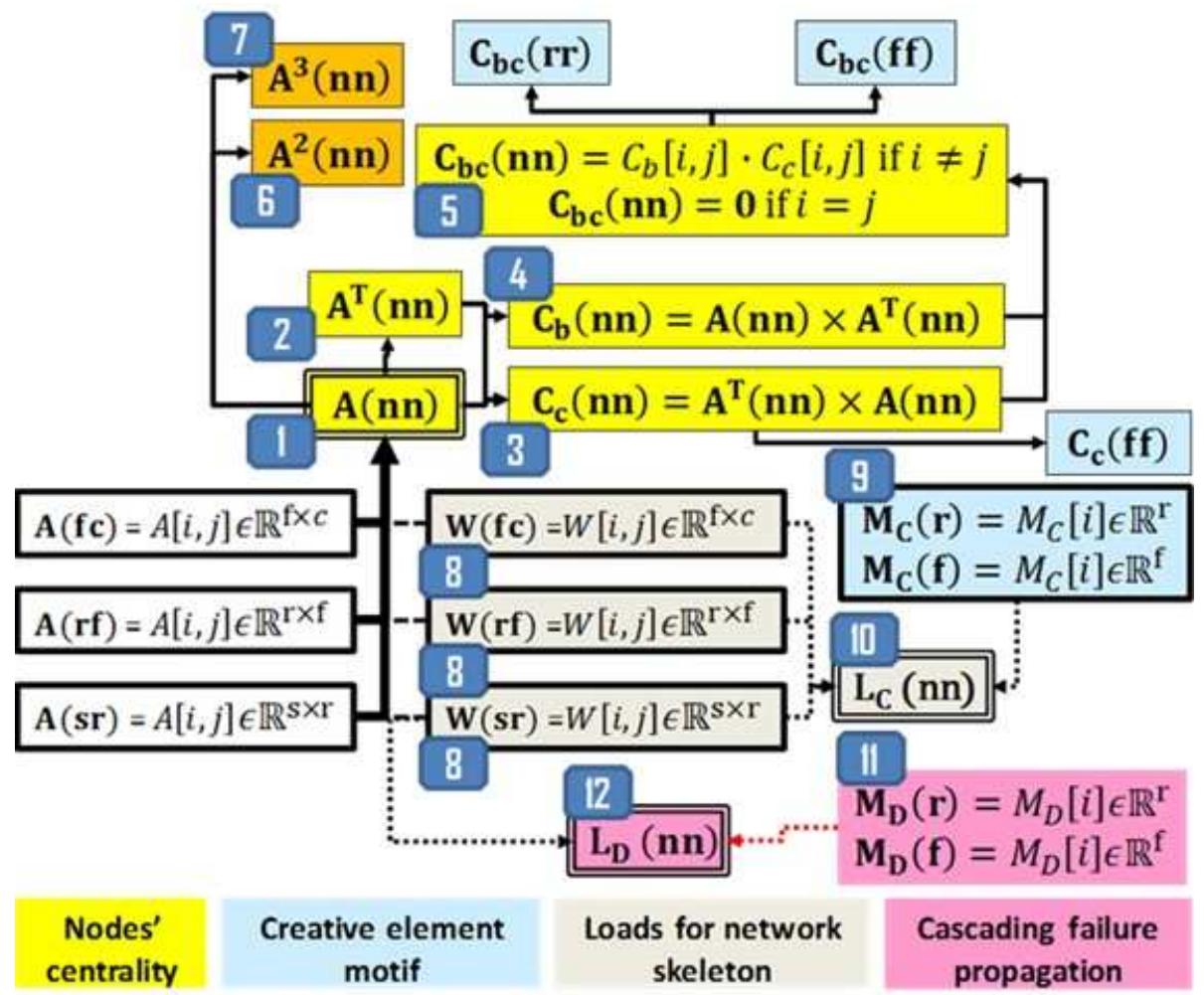

Fig. 4. Matrices for analysing an organization and their relationships

For mapping and quantifying the indirect and latent transversal dependences, first we build the co-citation matrix $-\mathbf{C}_{\mathbf{c}}(\mathbf{n n})$ - and the bibliographic coupling matrix $\mathbf{C}_{\mathbf{b}}(\mathbf{n n})$ - i.e. matrix product of $\mathbf{A}(\mathbf{n n})$ and its transposed and vice versa as per Eq. 43, 4-4. The results are seen in Fig. 5 for both the unweighted and weighted graphs.

The in-degree of the nodes in the matrix-diagonal of the co-citation matrix highlight the in-hubs of high in-degree in our organization (Fig. 5 top-left). Those in-hub finished products are magnifying the failure upstream when they become destabilized, since their in-links are not alternative ones (as in food webs), but are in strong sync. That is a typical phenomenon in a stretched and MRP-triggered supply chain, where the destabilized finished product in-hub pushes back all the non-missing resources (in relative excess vs the missing one) creating a multi-dimensional wave upstream. In the band-matrix part represented by sub-matrices $\mathbf{C}_{\mathbf{c}}(\mathbf{r r}) \mathbf{C}_{\mathbf{c}}(\mathbf{f f}) \mathbf{C}_{\mathbf{c}}(\mathbf{c c})$, on the other hand, we detect the number of common upstream-nodes of a node-pair in the same echelon, i.e. common finished products of two customers, common resources of two finished products and common suppliers of two resources. In other words, those are the bi-fans in DSN. The larger the number of common upstream-nodes in sub-matrix $\mathbf{C}_{\mathbf{c}}(\mathbf{f f})$ for given node-pair, the greater is the benefit of the creative element motif's solution designed by one of the authors. Therefore, sub-matrix $\mathbf{C}_{\mathbf{c}}(\mathbf{f f})$ is separately highlighted 
in Fig. 4 as one tool for detecting the creative element motifs in our organization. In our simplistic network F2 and F3 have more common resources than the other pairs as it is also seen in top-left matrix of Fig. 5.
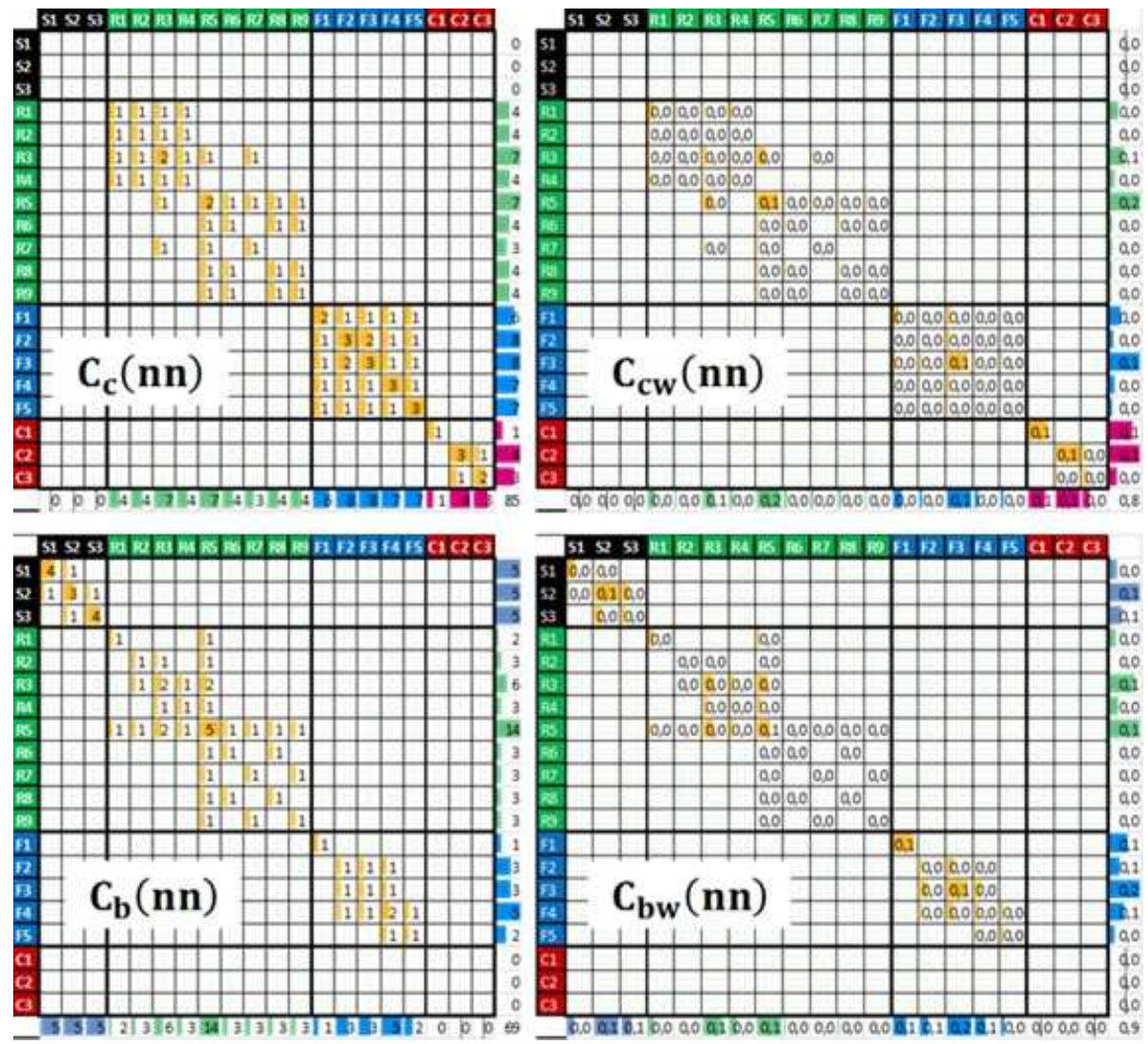

Fig. 5. Co-citation matrix $\mathbf{C}_{\mathbf{c}}(\mathbf{n n})$ without link weight (top-left) and $\mathbf{C}_{\mathbf{c w}}(\mathbf{n n})$ with link weight (top-right). Bibliographic coupling matrix $\mathbf{C}_{\mathbf{b}}(\mathbf{n n})$ without (bottom-left) and $\mathbf{C}_{\mathbf{b w}}(\mathbf{n n})$ with (bottom-right) link weight. In the diagonals of unweighted matrices (left) are seen the nodes' in-degree $\mathbf{C}_{\mathbf{c}}(\mathbf{n n})$ and out-degree $\mathbf{C}_{\mathbf{b}}(\mathbf{n n})$.

The bibliographic coupling matrix (Fig. 5 bottom-left) is created as matrix product of the adjacency matrix and its transpose (see Equation 4-4 in Fig 4.). The out-degrees of the nodes in the matrix-diagonal highlight the out-hubs. When an out-hub have low sales or consumption dollar-value, then we may ignore its network destabilization power, e.g. Zink ingots in Bowden-cable production at an automotive supplier. When such a node becomes unstable, it destabilizes its entire downstream out-component, which can be $60-80 \%$ of the organization. So it is pivotal to protect the out-hubs against upstream disruptions. In the band-matrix part we can detect the number of common 
downstream nodes of two nodes in the same echelon, i.e. common customers of two finished products, common finished products of two resources and common resources of two suppliers. The relationship between the nodes in pair may be competitive but also complementing (e.g. Rama Cube and Rama Brick margarines). The matrices of weighted network (Fig. 5, right) highlight that the inter-dependence between R3 and R5 became far stronger versus the non-weighted graph, due to the intensive use of them in high-runner F3 (common sink) and their common source/supplier S2.

The coexistence of common supplier/s and common finished product/s of a resource pair or the coexistence of common resource/s and common customer/s of a finished product pair results in strong emergent interdependence between the members of that node pair (Fig. 6). Such relationship rooted in the neighbouring echelons and creates the bi-parallel motifs. In case of larger number of common roots, the node pair may have several bi-parallels building stronger emergent interdependence, what plays crucial role during cascading failures.

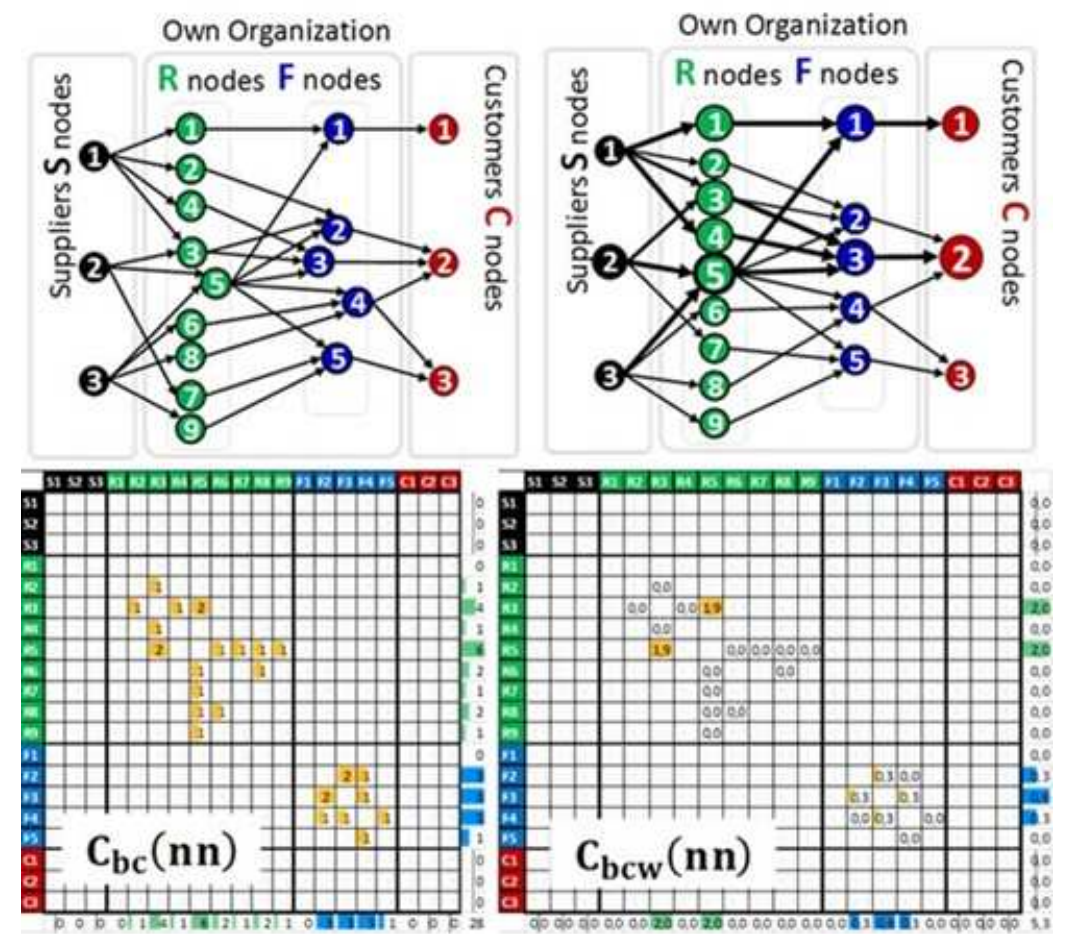

Fig. 6. Betweenness centrality matrix $\mathbf{C}_{\mathbf{b c}}(\mathbf{n n})$ based on bi-parallels for unweighted graph (left graph and matrix) and $\mathbf{C}_{\mathbf{b c w}}(\mathbf{n n})$ for weighted one (right graph and matrix)

We can visualize the number of bi-parallels through multiplying the similar entries of the co-citation and bibliographic coupling matrices as shown in Fig. 4 - Equation 4-5. That serves as a good betweenness centrality measure of the nodes in an organization - 
see Fig. 6. The material resource pairs with high value in $\mathbf{C}_{\mathbf{b c}}(\mathbf{r r})$ and the finished product pairs with high value in $\mathbf{C}_{\mathbf{b c}}(\mathbf{f f})$ are to be investigated for three purposes:

1. Those nodes can form value streams in our organization thanks to their high commonalities and connectedness

2. Those nodes with their high betweenness possess central role in failure propagation

3. A node-pair of a DNO and a CNO with high value in $\mathbf{C}_{\mathbf{b c}}(\mathbf{f f})$ matrix are excellent candidates for utilizing the creative element motif, especially protecting those PNO's which have also high value in $\mathbf{C}_{\mathbf{b c}}(\mathbf{f f})$ matrix with both DNO and CNO of that node-pair [5].

\section{Further potentials of the matrix approach}

Evidently, in the real life we also detect (limited) reverse flow of materials, e.g. finished products rejected by customers ( $\mathrm{C}$ to $\mathrm{F}$ ), in-progress returns in margarine production or in iron casting ( $\mathrm{F}$ to $\mathrm{R})$, rejected by our organization materials to our suppliers ( $\mathrm{R}$ to $\mathrm{S}$ ). In such cases the relevant directed links appear in the lower triangle part of our quadratic matrix in the relevant rectangular area of echelon pair also with positive number.

In demand-supply networks the directed paths are usually short, i.e. the nodes are in few steps distance from each other. Especially, in case of failure dispersion, when the directed links/edges turn to symmetrical, undirected. In other words, the failure advances both down-stream and up-stream in the network. Some material conversion and transport routes, on the other hand, may be well isolated from each other, what can be utilized in creating value streams through value stream mapping $[8,16]$. For defining the path length of 2,3 , etc. between nodes a raising of the quadratic matrix $\mathbf{A}(\mathbf{n n})$ to power of 2, 3, etc. is required according to the matrix operations of 6 and 7 in Fig 4 . If we include the mostly redundant men-nodes and machine-nodes then the interconnectedness increases radically and the number of independent paths from suppliers to customers will raise. Those links are mostly weak links - in some cases socalled creative links, i.e. the multi-skilled operators and multi-purpose machines with degenerative redundancy can work/be utilized in producing different finished products $[3,14,15]$. Thanks to mostly weak links of men-nodes and machine-nodes, those turn rarely to conduits for failure diffusion.

For modelling the propagation of the cascading failures our assumption is that symmetrical matrices need to be used as the failure can spread both upstream and downstream (Fig. 7). The emergent interdependence of the nodes through multiple bifans and bi-parallels can be considered in matrix models by adding the betweenness centrality values to the adjacency matrices $-\mathbf{C}_{\mathbf{b c}}(\mathbf{r r})$ and $\mathbf{C}_{\mathbf{b c}}(\mathbf{f f})$ respectively. We see high potential in adapting the traffic forecasting and traffic assignment approach [1], where the basic load through nodes and links could be represented by the echelon-level normalized weights of matrices $\mathbf{W}(\mathbf{s r}), \mathbf{W}(\mathbf{r f}), \mathbf{W}(\mathbf{f c})$, and by the vector matrices $\mathbf{M}_{\mathbf{C}}(\mathbf{r}), \mathbf{M}_{\mathbf{C}}(\mathbf{f})$ - delivered by Equation 3-6 in Fig. 3 and by Equations 4-8, 4-9, 4-10 in Fig. 4. In case of modelling the propagation of cascading failure in the organization, the meta-level irregularity of destabilized node is to be used $\mathbf{M}_{\mathbf{D}}(\mathbf{r}), \mathbf{M}_{\mathbf{D}}(\mathbf{f})$, while the links 
turn into strong transversal sync - Equation 3-5 in Fig. 3 and Equations 4-8, 4-11, 4-12 in Fig. 4.
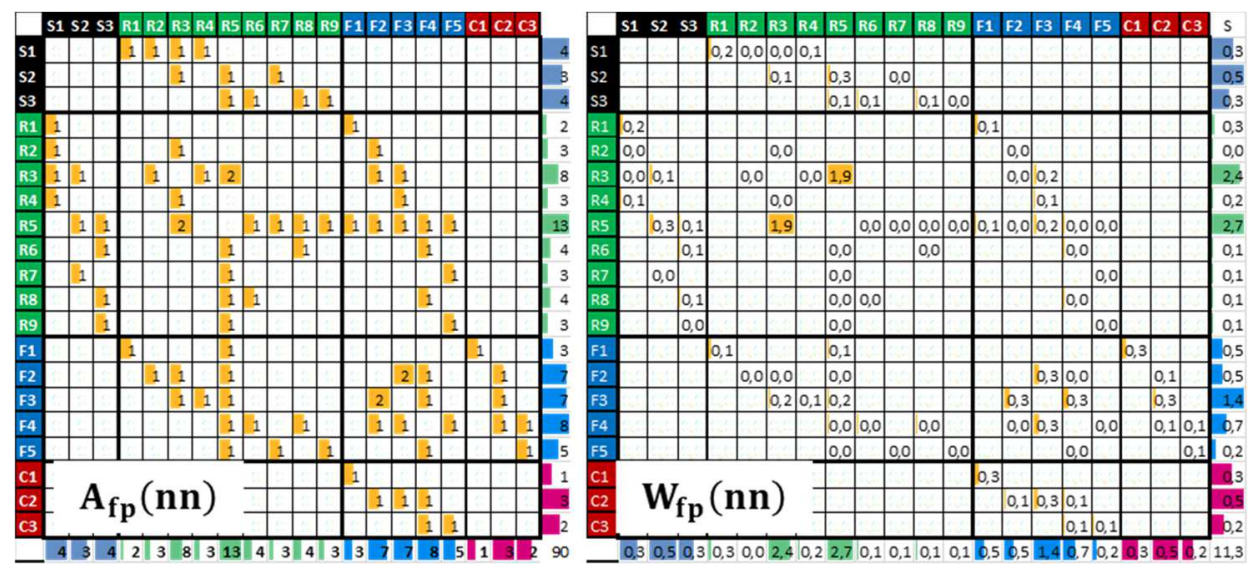

Fig. 7. Symmetrical adjacency matrices representing undirected network completed with $\mathbf{C}_{\mathbf{b c}}(\mathbf{r r})$ and $\mathbf{C}_{\mathbf{b c}}(\mathbf{f f})$ betweenness centrality figures for considering emergent interdependence. Unweighted on the left, weighted on the right.

\section{Conclusion}

The material conversion and transport route concept highlights the similarities and differences of DSN with other viable networked systems in biochemical kingdom. Structural similarities between DSN and biochemical networks lead to functional similarities as well. In our recent article we focused on identifying the supply chain specific aspects and their representation through the set of well-proven matrix analytical approaches [9]. First we have determined the adjacency matrix representation of an organization, i.e. nilpotent upper-triangle quadratic matrix with rectangular blocks of bipartite adjacent echelon-pairs. That matrix representation appears to be a powerful tool for making some typical network analytical operations. In the same time, we have given clear supply chain and operations management interpretations to those network analytical methods. It is crucial to determine what type of data are used for creating time related data sets. These latter serve the basis for creating basic matrices and more intricate derivatives to detect the main characteristics of the network topology and the diffusion of disruptions, failures of the organization. We see such investigations and calibrations as next steps in our research.

\section{References}

[1] Bakó A, Kádas S: A forgalomszétosztási feladat optimalizációs problémái. Alkalmazott Matematikai Lapok, Vol.7, pp. 107-137, 1981.

[2] Csermely P: Creative elements: network-based predictions of active centres in proteins and cellular and social networks. in Trends in Biochemical Sciences. Vol. 33, No. 12, pp. 569-576, 2008. 
[3] Csermely P: Weak Links. The Universal Key to the stability of Networks and Complex Systems. Springer, ISBN 978-3-540-31157-7, 2009.

[4] Csermely P, Korcsmáros T, Kiss HJ, London G, Nussinov R: Structure and dynamics of molecular networks: A novel paradigm of drug discovery.

Pharmacology \& Therapeutics, Vol. 138, pp. 333-408, 2013.

DOI: 10.1016/j.pharmthera.2013.01.016

[5] Fekete I, Hartványi T: Value chain stabilization with combined quantityirregularity graphs. in Tuncdan Baltacioglu et al eds. Value Chain Sustainability through Innovation and Design. Izmir, Izmir University of Economics Publication, pp. 189-199. ISBN 978-975-8789-50-4, 2013.

[6] Fekete I, Hartványi T: How can Network Science Help to Make an Organization Resilient and Successful. in GLOGIFT 15 Fifteenth Global Conference on Flexible Systems Management, Pune, India, 23-25 October 2015, pp. 1006-1017, ISBN 978-81-906294-9-2, 2015.

[7] Goldratt E, Cox J: The Goal. North River Press, Inc., ISBN 0-88427-061-0, 1992.

[8] Liker J: The Toyota Way. New York, McGraw-Hill. ISBN 0-07-139231-9, 2004.

[9] Newman MEJ: Networks. Oxford University Press Inc., Oxford, 2010.

[10] Palotai R, Csermely P: Network modules help the identification of key transport routes, signalling pathways in cellular and other networks. in Annalen der Physik 18, pp. 822-829, 2009.

DOI: $10.1002 /$ andp.200910378

[11] Snyder L, Zuo-Jun Max Shen: Fundamentals of supply chain theory. Hoboken, New Jersey, John Wiley \& Sons Inc., ISBN 978-0-470-52130-4, 2011.

[12] Supply Chain Council: Supply Chain Operations Reference (SCOR) model, Overview - Version 10.0, 2011.

[13] Vitasek KL, Manrodt KB, Kelly M: Solving the supply-demand mismatch. in Supply Chain Management Review, pp. 58-64, 2003.

[14] Whitacre J, Bender A: Degeneracy: a design principle for achieving robustness and evolvability. Journal of Theoretical Biology, Vol. 263, No. 1, pp. 143-152, 2013.

DOI: 10.1016/j.jtbi.2009.11.008

[15] Whitacre J: Degeneracy: a link between evolvability, robustness and complexity in biological systems. BMC Journal of Theoretical Biology and Medical Modelling, Vol. 7, No. 6, pp. 1-17, 2010.

DOI: $10.1186 / 1742-4682-7-6$

[16] Womack J, Jones D: Learning to see, 1999. 